\title{
EFFECTIVENESS OF AN INDOOR CYCLING PROGRAM IN IMPROVING THE PHYSICAL CONDITION OF YOUNG WOMEN
}

\author{
NATALIA CHUKHLANTSEVA
}

\author{
National University «Zaporizhzhia Polytechnic», Department of Physical Culture and Sport Management, \\ Ukraine
}

\author{
Mailing address: Natalia Chukhlantseva, National University «Zaporizhzhia Polytechnic», Department of Physical \\ Culture and Sport Management, 64 Zykovskogo Street, 69063 Zaporizhzhya, Ukraine, tel.: +380990888655, \\ e-mail: chuhnv@i.ua
}

\begin{abstract}
Introduction. Indoor cycling training programs at fitness clubs offer the possibility of optimizing the structure and amount of physical activity, which reduces the risk of disrupting the adaptive abilities of women's bodies. The main purpose of this study was to identify the effect of an indoor cycling program on the body composition and physical fitness of young women. Material and methods. Twenty-nine women (age $=29.31 \pm 3.40$ years, body weight $=70.71 \pm 6.15 \mathrm{~kg}$, and height $=169.83 \pm 3.17 \mathrm{~cm})$ took part in the study, participating in three classes per week for 16 weeks. Each class included three periods: the preparatory, main, and supporting periods. Body composition (waist circumference and body mass index), cardiorespiratory fitness (VO2max), motor fitness (balance), and musculoskeletal fitness (upper and lower body muscle strength and muscular endurance) were compared before the beginning of the study and after 10 and 16 weeks of training. Changes recorded in each variable over time were analyzed statistically using repeated measures methods. Results. Significant improvements in physical fitness were identified in the values of the body mass index (7.81\%; ES: 0.95, $\mathrm{p}=0.0001)$ and VO2max (12.51\%; ES: 1.02, $\mathrm{p}=0.0001)$. Moderate improvements were found in lower body muscle strength (11.13\%; ES: 0.66, $\mathrm{p}=0.0001)$ and waist circumference $(6.05 \%$, ES: $0.65, \mathrm{p}=0.0001)$. There was an increase in the strength of the muscles of the upper body (5.27\%; ES: 0.41, $\mathrm{p}=0.0001)$, muscular endurance (8.20\%; ES: 0.32, p = 0.0001), and balance (10.68\%; ES: 0.29, p = 0.003). Conclusion. Indoor cycling in a fitness club is an effective form of exercise for young women; it has targeted training effects on the body's functional systems, adaptive abilities, and physical fitness.
\end{abstract}

Key words: cycling, body composition, female, fitness

\section{Introduction}

A characteristic feature of the lifestyle of modern women is the insufficient amount of motor activity in combination with neuropsychic overload. The decrease in physical activity (PA) and the deterioration of physical fitness (PF) are associated with an increasing number of health problems that provoke metabolic disorders and degenerative changes in the muscular system, as well as accelerating the aging process [1]. The rational organization of PA, taking into account the age characteristics of women, suggests that they should undertake systematic physical training of varying intensity, which is diverse in terms of its effect on the body [2,3]. Health training at fitness clubs is carried out according to customer preferences and allows for optimizing the activity of the body's functional systems [4]. One type of programmed motor activity is indoor cycling, which is a form of group training on workout bikes. Indoor cycling is aerobic-oriented fitness with music, reliably simulating situations that occur in cycling. It has gained great popularity among men and women of different ages. Indoor cycling (IC) is practically universal cardiovascular training available to many people, regardless of their training level and weight. It does not overload the joints, trains the circulatory and respiratory organs, stimulates metabolism, increases endurance, as well as developing the leg, gluteus maximus, back, and abdominal muscles [5, $6,7]$. Rhythmic music encourages participants to move faster or slower, applying efforts that differ in magnitude, as well as making them feel greater pleasure and less fatigue [8]. Along with pedaling, IC programs use various movements engaging the muscles of the trunk and upper shoulder girdle, which contributes to increasing endurance, dynamic and static strength, speed, and the ability to quickly switch from one training mode to another. In studies on the effectiveness of cycling, it is emphasized that an adequate load can be provided due to a wide range of variations in pedal frequency and resistance level [9, 10]. Researchers have studied the features of thermoregulation and changes in lactate level after IC training, and the optimal parameters for the intensity of training were identified [11, 12, 13, 14]. The feasibility and effectiveness of applying IC programs in women of different ages, based on the optimization of physical activity and the selection of special exercises depending on the parameters of their functional state and the presence of diseases, have also been investigated $[15,16]$. The positive impact of IC training on cardiorespiratory fitness was revealed $[17,18]$. However, research into the effectiveness of IC classes in improving the physical fitness of young women is ignored by specialists. The investigation and improvement of the effectiveness of IC programs combining exercises of varying intensity with respect to enhancing the physical qualities of movement in women will allow not only to increase women's interest in these programs, but also help women achieve a new qualitative level of preparedness. The aim of the study was to experimentally substantiate the health-improving effects of IC classes by assessing their impact on body composition and physical fitness, namely the health-related components of the physical condition of young women, which should ultimately contribute to improving the effectiveness of health training. 


\section{Material and methods}

\section{Research participants}

This 16-week study involved twenty-nine women (age = $29.31 \pm 3.40$ years; body weight $=70.71 \pm 6.15 \mathrm{~kg}$; and height $=169.83 \pm 3.17 \mathrm{~cm}$; body mass index, $\mathrm{BMI}=24.72 \pm 2.07)$ who engaged in cycling at fitness club according to the proposed program under the guidance of an experienced fitness instructor. All participants were recreationally active, which means they performed physical activity of low intensity in their daily life and/or played sports from time to time, as well as having experience in fitness (they had been practicing it 1-3 times a week for at least a month). However, the participants had no experience in participating in systematic cycling workouts. Those who took part in the experiment had no cardiovascular, respiratory, metabolic, or musculoskeletal diseases or injuries which would restrict their ability to perform exercise. The subjects received instructions about the purpose of the study and the research methods. They were recommended to continue their regular diet and physical exercise throughout the study, but to refrain from consuming alcohol or caffeine-containing products and performing any type of exercise 48 hours before each trial. Written informed consent was obtained on all issues before conducting the study. All the tests were conducted in accordance with the Declaration of Helsinki. The study was approved by the Ethical Committee of the Department of Physical Culture and Sport Management at National University «Zaporizhzhia Polytechnic».

\section{Training program}

The participants attended 60-minute fitness cycling classes (including warm-up, conditioning, and cool-down) three times a week. The program was developed in accordance with the recommendations of the American College of Sports Medicine (ACSM) regarding the target heart rate which was to be achieved during different parts of the workout. Heart rate was measured using a Polar ${ }^{\circledast}$ heart rate monitor. The calculation of the intensity of the load was carried out according to the formula of Karvonen $($ Target Heart Rate $=[($ max HR - resting HR $) \times \%$ Intensity $]$ + resting HR). In the warm-up (8-10 min), the following exercises were performed: circular movements in the radiocarpal, elbow, and shoulder joints; body slopes sideways, abduction of hands for stretching the muscles; as well as stretching the quadriceps muscles of the thigh, the soleus muscles, the calf muscles, the gluteus maximus muscles, the gluteus deltoids, and the long and short flexors of the toes. The main part (40 min) included AeroFit PRO 9500B LCD stationary cycling exercises (AeroFit, FlowProducts, Netherlands). These were, for example:

1) imitation of riding on a flat road sitting: revolutions per minute (Rpm) - 70-90; hands in a narrow grip; one hand lies on the other and both lean on the handlebars in the center or hands shoulder-width apart; hands lie on the handlebars gripping them from above; resistance is low;

2) imitation of riding uphill sitting: Rpm - 60-80; landing average; hands shoulder-width apart gripping handlebars from above; resistance up to average or above average;

3) sprint (imitation of acceleration at a distance or at the finish): Rpm - maximum, landing low; starting sprint from a sitting position, rising on the saddle, and leaning forward to perform maximum acceleration; hands hold the bottom of the handlebars; resistance is large;

4) imitation of riding at a fast pace while standing (pedaling in standing position with hands gripping the top of the handlebars): Rpm - 80-100; landing standing; body is constantly engaged, standing on the pedals, not dropping into the saddle; holding hands wide at the top of the handlebars; resistance is moderate;

5) imitation of overcoming steep ascents, standing pedaling with hands gripping the bottom of the handlebars: Rpm - 50-60; pedaling standing on the pedals evenly without tilting the body and slightly transferring the weight of the body to the leg, which exerts pressure; hands are placed shoulder-width apart or slightly wider on top of the handlebars; body does not bend; body weight is used to increase the pressure on the pedal, and the body is shifted to the right and then to the left; the projection of the center of gravity is located above the pedals; hands hold onto the ends of the handlebars; resistance is medium or large;

6) imitation of overcoming steep ascents, standing pedaling with hands gripping the bottom of the handlebars: Rpm 50-60; pedaling while standing leaning forward; the center of gravity of the body shifts towards the handlebars; hands resting on the ends of the handlebars, slightly bent at the elbows; using hands as an additional point of support; hands increase the pressure on the pedals, thereby increasing the efficiency of using their motor potential; resistance is medium or large;

7) imitation of overcoming obstacles or driving on a rough road: Rpm - 80-100; driving with a change of position (then standing up while sitting); resistance is small or moderate.

In the cool-down part (8-10 min), breathing exercises and stretching exercises were performed, the purpose of which was to smoothly and slowly stretch the main muscle groups, such as the muscles of the back and shoulder girdle, the quadriceps muscles, the neck muscles, the pectoral muscles, the lumbar muscles, or the gastrocnemius muscles. Each exercise was performed no more than 10-30 seconds before the feeling of tension occurred. Static stretching exercises were performed in a standing position, with support or thrust on the bike. During the exercises, appropriate music was used according to different levels of intensity.

The 16-week program of cycling included three periods: the preparatory ( 3 weeks), main ( 8 weeks), and supporting ( 5 weeks) periods. The purpose of the preparatory period was the training and improvement of work techniques on the exercise bike. The intensity of the load in this period corresponded to the first training zone and ranged from 130 to $150 \mathrm{bpm}$, that is moderate intensity. The purpose of the main cycling training period was the implementation of modes of operation that ensure the improvement of aerobic-anaerobic and anaerobic performance; the loads in this period corresponded to the second training zone (from 150 to $180 \mathrm{bpm}$ ) and the third zone (more than $180 \mathrm{bpm}$ ). The duration of the main part increased from 30 to 35 minutes, and the intensity increased from $40-60 \%$ to 50 $85 \%$ of HR max; moreover, new exercises were added. In order to develop their speed abilities, the subjects performed sprints in the standing position. Balance was improved by implementing exercises concentrating on the work of one leg while standing, riding while standing with one arm on the handlebars, as well as alternating sitting and standing ("jumping"). Continuous exercise of varied intensity was predominantly used.

In the third period of preparation, to maintain the achieved level of physical condition, $80 \%$ of the time, the resistance was above average, with a pulse rate of $150 \mathrm{bpm}$ and above; the duration of the main part was increased to 40 minutes; and interval training workouts were used. In order to develop muscle strength, we increased resistance to $90 \%$ of a subject's personal maximum. Speed was improved by means of sitting, standing, and standing uphill sprints. Coordination was developed by 
implementing riding in a standing position without the hands supported on the handlebars, alternating sitting and standing, and alternating standing and standing uphill.

\section{Procedure}

We measured the subjects' anthropometric indices (height and weight) and assessed health-related physical fitness components, which were evaluated at the beginning of the study and after 10 and 16 weeks of intervention. All measurements and evaluations were carried out by the researcher at the fitness club. We measured body weight $(\mathrm{kg})$, height $(\mathrm{m})$, and waist circumference $(\mathrm{cm})$ with the use of a mechanical stadiometer, weights, and measuring tape, according to the standard conditions of anthropometric measurements. BMI was calculated based on height and weight $\left(\mathrm{kg} \cdot \mathrm{m}^{2}\right)$. To assess physical fitness $(\mathrm{PF})$, we used several tests included in the ALPHA-FIT test battery, a detailed description of which is presented in the literature [19]. The motor fitness index was assessed based on the totals of the one-leg stand test to measure balance, whereas musculoskeletal fitness was evaluated on the basis of the results of the hand grip, jump-and-reach, and modified push-up tests. To appraise the dynamics of the cardiorespiratory fitness (VO2max), we determined the OwnIndex for each subject using a Polar V800 heart rate monitor, following the recommendations of Polar [20, 21]. Before the test, the subject was asked to lie on her back for about 5 minutes. The heart rate at rest was constantly recorded during this rest period. So as to run the Polar fitness test, we recorded the subject's parameters (age, gender, height, and weight) onto the Polar watch. After starting the test, the participant remained in place, relaxing, without moving or communicating with others, for a 5-minute evaluation, while the Polar watches measured heart rate variability during rest. Progress was visible on the clock screen, and after the test was completed, the VO2max value $\left(\mathrm{ml} \cdot \mathrm{kg}^{-1} \cdot \mathrm{min}^{-1}\right)$ was recorded. Testing was carried out strictly after a day of rest.

\section{Statistical analysis}

The research results were processed using SPSS version 22.00 software (StatSoft Inc., Tulsa, OK, USA). The significance level was set at $\mathrm{p} \leq 0.05$. All the data were presented as means with standard deviations (SDs). To assess the nature of the cumulative distribution of the sample data, we used the Kolmogorov-Smirnov test. In all cases, the distribution of signs corresponded to the law of normal distribution; therefore, in the statistical analysis of each variable, changes over time were ana- lyzed with Bonferroni's multiple comparison test and the linear trend test. They were applied if the assumption of sphericity was violated. Measures of the effect size (ES) for differences were calculated by dividing the mean difference by the standard deviation of the pre-training measurement. The magnitude of the effect size was classified according to the following criteria: $0.2<\mathrm{d}<0.5$ was considered small ES, $0.5<\mathrm{d}<0.8$ represented medium ES, and $d>0.8$ constituted large ES [22].

\section{Results}

The results of measuring the body composition and physical fitness indicators of women who participated in the experiment $(n=29)$ before, during, and after the experiment are presented in Table 1.

According to the results obtained for the anthropometric variables presented in Table 1 , it can be noted that after 10 weeks of intervention, the proposed indoor cycling program contributed to positive changes at a high level of statistical significance. There was a decrease of waist circumference of $4.19 \%$, the average difference between the values being $3.19 \mathrm{~cm}\left(\mathrm{~F}_{1,28}=59.265\right.$, $\mathrm{p}$ $<0.0001)$. After 16 weeks of intervention, the decrease observed for this variable was already $6.05 \%$, and the average difference between the values reached $4.61 \mathrm{~cm}\left(\mathrm{~F}_{2,56}=66.947, \mathrm{p}<0.0001\right)$. An analysis of the changes in the results of intermediate BMI testing similarly indicates a positive impact of our program on this variable. The average difference in BMI after 10 weeks of the experiment was $1.39 \mathrm{~kg} \cdot \mathrm{m}^{2}$, which equaled $5.68 \%\left(\mathrm{~F}_{1,28}=\right.$ 3999.757, $\mathrm{p}<0.0001)$. At the end of the study, the decrease in this variable was already $7.81 \%$, the average difference between the values being $1.91 \mathrm{~kg} \cdot \mathrm{m}^{2}\left(\mathrm{~F}_{1,28}=3973.516, \mathrm{p}<0.0001\right)$.

Some indicators of physical fitness over a 10 -week period also improved. The muscle strength of the upper body, according to the results of the hand grip test, increased by $3.82 \%\left(\mathrm{~F}_{1,28}\right.$ $=463.369, \mathrm{p}<0.0001)$, the average difference in values being $1.50 \mathrm{~kg}$. After 16 weeks of intervention, in comparison with the initial data, the decrease in this variable was already $5.27 \%$, and the average difference between the values was $2.07 \mathrm{~kg}\left(\mathrm{~F}_{2,56}=\right.$ 307.185, $\mathrm{p}<0.0001)$. It is worth stressing that the effect of the indoor cycling program on the indicator of muscle strength of the lower body was found to be more pronounced: the average difference between the results of the jump-and-reach test before and after 10 weeks of training was $2.52 \mathrm{~cm}$, which amounted to $10.15 \%\left(F_{1,28}=55.182, p<0.0001\right)$. At the end of the study, the increase in jump height was already $11.13 \%$, the average difference

Table 1. Evaluation of body composition, motor, musculoskeletal and cardiorespiratory fitness indicators in response to cycling training. Mean ( \pm SD) results are given.

\begin{tabular}{|c|c|c|c|c|c|c|}
\hline Indicators measured & Before training & $\begin{array}{l}\text { After } 10 \text { weeks of } \\
\text { training }\end{array}$ & $\begin{array}{l}\text { After } 16 \text { weeks of } \\
\text { training }\end{array}$ & $\begin{array}{c}\text { P-value } \\
(0-16)\end{array}$ & $\begin{array}{l}\text { Linear trend } \\
(0-16)\end{array}$ & $\begin{array}{l}\text { Absolute difference } \\
\text { (ES) }\end{array}$ \\
\hline Waist circumference $[\mathrm{cm}]$ & $76.00(7.59)$ & $72.87(6.89)^{*}$ & $71.46(6.45)$ & 0.0001 & 0.0001 & $-4.61(0.65)$ \\
\hline $\mathrm{BMI}\left[\mathrm{kg} \cdot \mathrm{m}^{2}\right]$ & $24.52(2.09)$ & $23.12(1.98)^{*}$ & $22.60(1.93)$ & 0.0001 & 0.0001 & $-1.91(0.95)$ \\
\hline One-leg stand [s] & $46.37(24.13)$ & $47.17(23.30)$ & $51.33(21.5)$ & 0.003 & 0.002 & $4.96(0.29)$ \\
\hline Hand grip [kg] & $39.23(5.16)$ & $40.73(5.22)^{*}$ & $41.30(5.07)$ & 0.0001 & 0.0001 & $2.07(0.41)$ \\
\hline Jump-and-reach [cm] & $24.79(4.39)$ & $27.31(1.02)^{*}$ & $27.55(5.83)$ & 0.0001 & 0.0001 & $2.76(0.66)$ \\
\hline Modified push-up [total number] & $10.51(2.75)$ & $10.62(2.89)$ & $11.38(2.78)$ & 0.0001 & 0.0001 & $0.86(0.32)$ \\
\hline V02 $\mathrm{max}\left[\mathrm{ml} \cdot \mathrm{kg}^{-1} \cdot \mathrm{min}^{-1}\right]$ & $33.67(3.89)$ & $36.32(4.39)^{*}$ & $37.88(4.37)$ & 0.0001 & 0.0001 & $4.21(1.02)$ \\
\hline
\end{tabular}

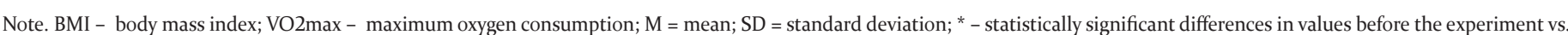
after 10 weeks of training at the level of $\mathrm{p}<0.05$; ES - effect size (Cohen's $d$ ). 
between the values being $2.76 \mathrm{~cm}\left(\mathrm{~F}_{1,28}=50.349, \mathrm{p}<0.0001\right)$. These data are very important because an increase in muscle strength contributes to a greater efficiency of daily activities.

It should be noted that after 10 weeks of training, cardiorespiratory fitness improved significantly: the average difference in VO2max was $2.65 \mathrm{ml} \cdot \mathrm{kg}^{-1} \cdot \mathrm{min}^{-1}$, which corresponds to $7.87 \%$ $\left(F_{1,28}=55.697, p<0.0001\right)$. At the end of the study, in comparison with the initial data, an increase in this variable of $12.51 \%$ was observed, the average difference between the values being $4.21 \mathrm{ml} \cdot \mathrm{kg}^{-1} \cdot \mathrm{min}^{-1}\left(\mathrm{~F}_{1,38}=31.761, \mathrm{p}<0.0001\right)$.

Interestingly, after 10 weeks of intervention, only a slight increase of the balance and endurance of the body muscles was revealed. Table 1 also shows data that indicate that the growth of the balance indicator, according to the results of the oneleg stand test, averaged $0.79 \mathrm{~s}$, that is $1.72 \%\left(\mathrm{~F}_{1,28}=4.503, \mathrm{p}=\right.$ 0.127 , which means that these changes were not statistically significant. Over the following weeks of training under the proposed program, the results eventually increased by $4.95 \mathrm{~s}$, which amounted to $10.68 \%\left(\mathrm{~F}_{1,31}=9.817, \mathrm{p}=0.003\right)$. The smallest improvement was found in the modified push-up test. The average difference between the initial and intermediate testing values was only 0.10 push-up or $0.99 \%\left(\mathrm{~F}_{1,28}=0.592\right.$, $\left.\mathrm{p}=1.00\right)$, meaning that these changes were not statistically significant. After 16 weeks of intervention, in comparison with the initial data, the increase in this variable was already $8.20 \%$, the average difference between the values being 0.86 push-up $\left(\mathrm{F}_{2,56}=24.844\right.$, $\mathrm{p}<0.0001)$.

\section{Discussion}

The variety and constant updating of the programs and the emotional states generated by indoor cycling classes contribute to their great popularity among fitness club clients [4]. The purpose of this study was to examine the effects of an experimental cycling program on health-related and skill-related components of physical fitness in young women. Overall, the results confirm our hypothesis that a 16-week aerobic cycling program contributes to a marked improvement in physical fitness (PF). The study revealed significant improvements in BMI and maximum oxygen consumption. Moderate improvements were found in lower muscle strength and waist circumference.

Together with improved physical fitness, the optimization of anthropometric indices was one of the goals of cycling training, since abdominal obesity, measured based on waist circumference (WC) in combination with BMI, reliably predicts possible health risks. It is known that cycling training is an effective tool for optimizing body composition and reducing BMI [23, 24]. Our results show that cycling training carried out according to the proposed program, without any restrictions in the diet, is effective for weight loss and stimulates decreases in WC in young women (of $6.05 \%$ on average). This corresponds with the results obtained by other researchers [18, 24]; for example, Kaya et al. [24] recorded a significant decrease in BMI after 3 weeks of cycling training. This is probably due to the fact that exercise in the aerobic and anaerobic regimes has a positive effect on muscle mass and improves basic metabolism. The decrease in BMI by $7.81 \%$ can be explained by the fact that the training took place in the high-power zone with a large amount of oxygen consumption and that several large muscle groups were involved in the exercises. The data obtained is inconsistent with the data in studies where no change or less significant change was observed in this indicator $[2,17]$. These discrepancies can be explained by the differences in the length of the intervention period (from 8 to 12 weeks), the intensity and type of exercise, the categories of participants under study, their number, their age, as well as the presence or absence of obesity or chronic diseases. Studies conducted earlier show that the exercise program is more effective when a healthy diet is observed, and positive changes in body composition are more pronounced in such situations compared to the implementation of diet or physical activity only [25, 26].

Indicators of muscle strength of the lower body (measured by jumping up) and balance (according to the results of the one-leg stand test) improved by $11.13 \%$ and $10.68 \%$, respectively, which indicates that the experimental program has great potential and should be used in fitness classes to increase the level of physical fitness. It is known that the ability to maintain the balance of the body in difficult postural conditions is determined by the strength of the back muscles, the rectus abdominis muscles, and the leg muscles (biceps femoris, ankle joint muscles, and knee and hip joint muscles). All of them were actively involved in the work during the exercise, performing the flexion and extension of the hip, knee, and ankle joints. Our results confirm the findings of previous studies and complement data showing that postural stability is an indicator of the adaptive capacity of the organism and its functional systems [15]. The proposed exercises also contributed to the development of muscular endurance due to the continuous inclusion of the abdominal muscles and the back muscles in the work (for example, keeping the body in the correct position). Exercises performed in the standing position, bearing in mind that the participants lacked experience in IC exercise, were a means of developing coordination - maintaining balance, adopting a correct starting position, and using correct technique in riding a bike. It was revealed that static balance and muscle strength are related to each other in young adult women, and the success achieved in one variable after training is interconnected with changes in the indicators of the other variable. The final results of the study revealed an increase in hand muscle strength and also in the total strength of the arm muscles, which is necessary to perform many daily functions. The increase in the results of the hand grip test of $5.27 \%$ and in the modified push-up test of $8.20 \%$ indicates a greater effectiveness of training with resistance above the average of the individual maximum in women, in which, in addition to the leg muscles, the oblique muscles of the body are actively involved (the body tilts to the right and left to help overcome resistance), as well as the muscles of the arms and chest, because the pressure on the handlebars increases (especially when imitating driving uphill while standing with high resistance).

The results of this study show that cycling workouts are effective in improving cardiorespiratory fitness. The magnitude of the change in VO2max in the current study (by $12.51 \%$ ) is comparable with data from previous studies [2, 14, 23, 24, 25, 27]. For example, 8-week cycling sessions of moderate and high intensity contributed to an increase of $11-12 \%$ in $\mathrm{VO} 2 \mathrm{max}$ in female students aged 20-21 years [2]. The results of another study also showed that VO2max increased after participating in 8 weeks of cycling classes (three times a week) [18]. The changes in this indicator during the experiment confirmed that the classes increased the women's aerobic capabilities, meaning that they enhanced the body's oxygen transport capabilities and the aerobic capabilities of the skeletal muscles that utilize oxygen in oxidative processes of energy production, and the ability of the muscles to oxidize carbohydrates and especially fats was improved.

This research is one of few studies dedicated to studying the effect of IC programs on the physical fitness of young, recreationally active women. An attempt was made to explore this practice in a real context, analyzing the changes in the indica- 
tors studied, which is one of the strengths of our study. Nevertheless, we note some limitations of our study. Despite the fact that the participants of the experiment were not asked to adhere to special diets, we cannot exclude the possibility that some had changed their diet. Since IC programs are very diverse, we do not claim that the training program we have offered is superior to others. Moreover, the results obtained cannot be compiled with data reported for other groups in the population (men, children, and the elderly). However, this study provides opportunities for future research, in which other assessment methods can be used, in addition to ours, to refine our results.

\section{Conclusions}

Systematic IC exercise is effective in maintaining the optimal level of indicators characterizing the state of health, especially body composition and physical fitness. They enhance adaptive mechanisms, as well as improving metabolic processes and blood supply to tissues and organs, which positively affects participants' general physical condition, well-being, and efficiency. The results of this study confirm our basic hypothesis that IC is an effective tool for improving cardiometabolic health and physical fitness in young women when performed in a group workout format at a fitness club. Continued, systematic participation in IC classes, optimally selected individual loads, the addition of standard pedaling, and the interchangeable use of a variety of techniques increase the ability to resist fatigue and improve performance. Such training programs can be an alternative for people who are not attracted to traditional aerobics, and the results of the study may be relevant for fitness clubs and sports instructors who are starting to teach fitness classes.

\section{Literature}

1. Piercy K.L., Troiano R.P., Ballard R.M., Carlson S.A., Fulton J.E., Galuska D.A. et al. (2018). The physical activity guidelines for Americans. JAMA 320(19), 2020-2028. DOI: 10.1001/jama.2018.14854.

2. Mazurek K., Zmijewski P., Krawczyk K., Czajkowska A., Kęska A., Kapuściński P. et al. (2016). High intensity interval and moderate continuous cycle training in a physical education programme improves health-related fitness in young females. Biology of Sport 33(2), 139-144. DOI: 10.5604/20831862.1198626.

3. Nowak M. (2008). Impact of recognized by women health and physical-fitness-related values on the length of their physical activity. Polish Journal of Sport and Tourism 15(4), 174-183.

4. Moving communities: Active leisure trends 2018. (n.d.). Retrieved from http://research.ukactive.com/wp-content/ uploads/2018/06/Moving-Communities-Active-LeisureTrends-2018.pdf.

5. Piacentini M.F., Gianfelici A., Faina M., Figura F., Capranica L. (2009). Evaluation of intensity during an interval Spinning ${ }^{\circledR}$ session: A field study. Sport Sciences for Health 5(1), 29-36. DOI: 10.1007/s11332-009-0073-y.

6. López-Miñarro P., Rodríguez J.M. (2010). Heart rate and overall ratings of perceived exertion during Spinning ${ }^{\circledR}$ cycle indoor session in novice adults. Science $\mathcal{E}$ Sports 25(5), 238-244. DOI: 10.1016/j.scispo.2009.11.003.

7. de Melo Dos Santos R., Costa F., Saraiva T.S., Callegari B. (2017). Muscle fatigue in participants of indoor cycling. Muscles, Ligaments and Tendons Journal 7(1), 173-179. DOI: 10.11138/mltj/2017.7.1.173.
8. Shaulov N., Lufi D. (2009). Music and light during indoor cycling. Perceptual and Motor Skills 108(2), 597-607. DOI: 10.2466/pms.108.2.597-607.

9. Muyor J.M. (2013). Exercise intensity and validity of the ratings of perceived exertion (Borg and OMNI Scales) in an indoor cycling session. Journal of Human Kinetics 39(1), 93101. DOI: 10.2478/hukin-2013-0072.

10. Barbado C., Foster C., Vicente-Campos D., López-Chicharro J. (2017). Exercise intensity during indoor cycling. Revista Internacional De Medicina Y Ciencias De La Actividad Física Y Del Deporte 67(2017). DOI: 10.15366/rimcafd2017.67.006.

11. Ipekoğlu G., Baynaz K., Mor A., Acar K., Arslanoğlu C., Arslanoğlu E. (2018). Examining lactate changes during high intensity Spinning ${ }^{\circledR}$ training. Universal Journal of Educational Research 6(6), 1260-1263. DOI: 10.13189/ ujer.2018.060617.

12. Hazelhurst L.T., Claassen N. (2006). Gender differences in the sweat response during spinning exercise. The Journal of Strength and Conditioning Research 20(3), 723. DOI: 10.1519/18155.1.

13. Caria M.A., Tangianu F., Concu A., Crisafulli A., Mameli O. (2007). Quantification of Spinning ${ }^{\circledR}$ bike performance during a standard 50-minute class. Journal of Sports Sciences 25(4), 421-429. DOI: 10.1080/02640410600718533.

14. Battista R.A., Foster C., Andrew J., Wright G., Lucia A., Porcari J.P. (2008). Physiologic responses during indoor cycling. The Journal of Strength and Conditioning Research 22(4), 1236-1241. DOI: 10.1519/jsc.0b013e318173dbc4.

15. Vilarinho R., Souza W.Y., Rodrigues T.C., Ahlin J., Junior D.G., Barbosa F. (2009). Effects of indoor cycling in body composition, muscular endurance, flexibility, balance and daily activities in physically active elders. Fitness $\mathcal{E}$ Performance Journal 8(6), 446-451. DOI: 10.3900/fpj.8.6.446.e.

16. Kim H., Kim H., Park M., Kang H. (2015). A comparative study on cycling in relation to the physical balance, muscle strength, self-esteem and depression of elderly women. Indian Journal of Science and Technology 8(S1), 169. DOI: 10.17485/ijst/2015/v8isl/57901.

17. Bianco A, Bellafiore M, Battaglia G, Paoli A, Caramazza G, Farina F. et al. (2010). The effects of indoor cycling training in sedentary overweight women. Journal of Sports Medicine and Physical Fitness 50, 159-165.

18. Faulkner S.H., Pugh J.K., Hood T.M., Menon K., King J.A., Nimmo M.A. (2015). Group studio cycling; an effective intervention to improve cardio-metabolic health in overweight physically inactive adults. Journal of Fitness Research 4(2), 16-25.

19. ALPHA-FIT Test Battery. (2009). Retrieved March 21, 2019, from http://www.ukkinstituutti.fi/filebank/500-ALPHA FIT_Testers_Manual.pdf.

20. Cooper K.D., Shafer A.B. (2019). Validity and reliability of the Polar A300's fitness test feature to predict VO2max. International Journal of Exercise Science 12(4), 393-401.

21. User manual (2018), "V800 Polar", available at: https://support.polar.com/e_manuals/V800/Polar_V800_user_manual_Russian/Content/Printed\%20documentation/manual.pdf.

22. Cohen J. (1988). Statistical power analysis for the behavioral sciences (2nd ed.). Hillsdale, NJ: Lawrence Erlbaum Associates.

23. Valle V.D., Mello D.D., Fortes M.D., Dantas E. (2009). Effects of indoor cycling associated with diet on body com- 
position and serum lipids. Biomedical Human Kinetics 1(1). DOI: 10.2478/v10101-009-0004-z.

24. Kaya F., Nar D., Erzeybek M.S. (2018). Effect of spinning cycling training on body composition in women. Journal of Education and Training Studies 6(4), 154. DOI: 10.11114/jets. v6i4.3028.

25. Higgins S., Fedewa M.V., Hathaway E.D., Schmidt M.D., Evans E.M. (2016). Sprint interval and moderate-intensity cycling training differentially affect adiposity and aerobic capacity in overweight young-adult women. Applied Physiology, Nutrition, and Metabolism 41(11), 1177-1183. DOI: 10.1139/apnm-2016-0240.
26. Yoon J. G., Kim S. H., Rhyu H. S. (2017). Effects of 16-week spinning and bicycle exercise on body composition, physical fitness and blood variables of middle school students. Journal of Exercise Rehabilitation 13(4), 400-404. DOI:10.12965/jer.1735052.526.

27. Klika R.J., Alderdice M.S., Kvale J.J., Kearney J.T. (2007). Efficacy of cycling training based on a power field test. The Journal of Strength and Conditioning Research 21(1), 265-269. DOI: 10.1519/00124278-200702000-00047.

Submitted: July 1, 2019.

Accepted: October 24, 2019. 